\title{
Multimodality imaging in Europe: a survey by the European Society of Radiology (ESR) and the European Association of Nuclear Medicine (EANM)
}

\author{
European Society of Radiology (ESR) • \\ European Association of Nuclear Medicine (EANM)
}

Received: 16 October 2009 /Accepted: 28 October 2009/Published online: 28 January 2010

(C) European Society of Radiology 2010

\begin{abstract}
Purpose Multimodality imaging represents an area of rapid growth with important professional implications for both radiologists and nuclear medicine physicians throughout Europe. As a preliminary step for future action aimed at improving the quality and accessibility of PET-SPECT/CT multimodality imaging practice in Europe, the European Society of Radiology (ESR) and the European Association of Nuclear Medicine (EANM) performed a survey among the individual memberships of both societies to obtain information on the status of multimodality imaging in their facilities and their future visions on training for combined modalities. Methods A questionnaire was forwarded to all individual members of ESR and EANM. The main subject matter of the questionnaire related to: (1) study performance, current procedures and current equipment, including its supervisory personnel at respondents' individual facilities, and (2) vision of future practice, performance and the potential for combined interdisciplinary viewing and training for future professionals. Results The reporting and the billing procedures of multimodality imaging studies are very heterogeneous in European countries. The majority of the members of both societies believe that the proportion of PET/CT conducted
\end{abstract}

European Society of Radiology $(\bowtie)$

Neutorgasse 9,

1010 Vienna, Austria

e-mail: communications@myesr.org

European Association of Nuclear Medicine $(\triangle)$

Hollandstrasse 14 / Mezzanine,

1020 Vienna, Austria

e-mail: office@eanm.org as a full diagnostic CT with contrast enhancement will increase over time. As expected, 18F-FDG is the most used PET tracer for clinical applications. The large majority of respondents were in favour of an interdisciplinary training programme, being developed on a European level together by ESR and EANM and the respective sections of the European Union of Medical Specialists.

Conclusion The results of this survey show that there is wide heterogeneity in the current practice of multimodality imaging in Europe. This situation may limit the full potential and integration of multimodality imaging within the clinical arena. There is a strong desire within both specialties for the development of interdisciplinary training to address some of these issues.

Keywords Multimodality imaging systems · European Society of Radiology · European Association of Nuclear Medicine

\section{Introduction}

Multimodality imaging represents an area of very rapid growth with important professional implications. It may be anticipated that combined functional and anatomical imaging with positron emission tomography (PET)/computed tomography (CT), single-photon emission computed tomography (SPECT)/CT and even PET/magnetic resonance imaging will play a central part in diagnostic imaging of the near future. In 2007, the European Society of Radiology and the European Association of Nuclear Medicine published a document entitled "White Paper of the European Society of Radiology 
Table 1 Respondents by country.

\begin{tabular}{lllll}
\hline ESR & & & EANM & \\
\cline { 1 - 2 } \cline { 5 - 5 } Country & Number & & Country & Number \\
\hline Italy & 219 & & France & 40 \\
Germany & 179 & & Italy & 32 \\
Spain & 158 & & The Netherlands & 26 \\
United Kingdom & 89 & & Germany & 24 \\
France & 74 & & Turkey & 21 \\
The Netherlands & 64 & & Belgium & 16 \\
Austria & 57 & & Spain & 13 \\
Belgium & 52 & & United Kingdom & 12 \\
Poland & 51 & & Greece & 11 \\
Greece & 50 & & Portugal & 9 \\
Others & 498 & & Others & 146 \\
Total & 1,491 & & Total & 350 \\
\cline { 1 - 1 } & & & &
\end{tabular}

(ESR) and the European Association of Nuclear Medicine (EANM) on Multimodality Imaging" [1, 2]. This paper sets out the positions and aspirations of ESR and EANM to work together on an equal and constructive basis for the future benefit of both specialties on which to build. To provide a database for these efforts, ESR and EANM performed a survey among the individual memberships of both societies. The aim was to obtain information on the status of multimodality imaging equipment and procedures throughout Europe, and to identify any future visions on training for combined modalities amongst respondents.

\section{Methods}

A questionnaire was forwarded to all individual members of ESR and EANM. The questions posed included: (1) aspects of current practice procedures and equipment at the individual respondent's facility and (2) vision on cooperative interdisciplinary training for the future. The questionnaire structure is reported in the Appendix.

Table 2 Respondents by age group.

\begin{tabular}{lll}
\hline Age group (years) & ESR $(\mathrm{n}=1,491)$ & EANM $(\mathrm{n}=350)$ \\
\hline $21-30$ & $8 \%$ & $3 \%$ \\
$31-40$ & $30 \%$ & $23 \%$ \\
$41-50$ & $32 \%$ & $38 \%$ \\
$51-60$ & $23 \%$ & $24 \%$ \\
$61+$ & $7 \%$ & $12 \%$ \\
\hline
\end{tabular}

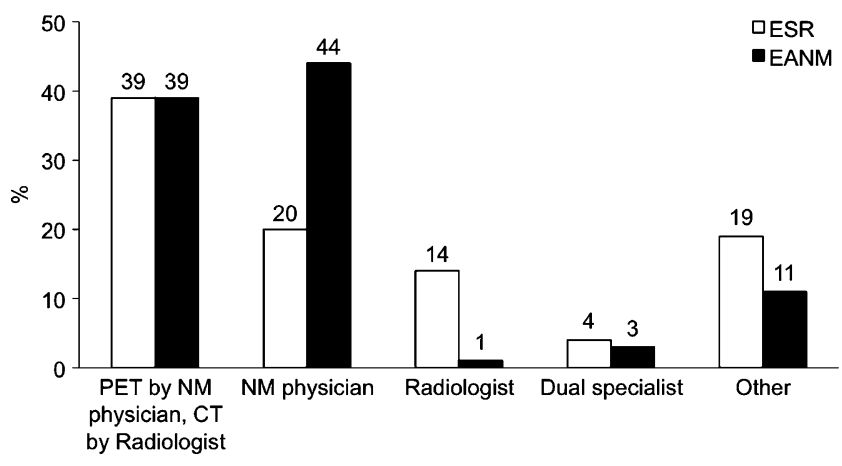

Fig. 1 Answers to question 1 of the survey, "In your facility, PET/CT results are reported by..."

\section{Results}

A total of 1,841 replies were received: 1,491 from ESR members (from 38 European countries) and 350 from EANM members (from 39 countries). The answers to the questionnaires formed the basis of the currently reported analysis. The respondents by country and age group are reported in Tables 1 and 2, respectively. The reporting (Fig. 1) and the billing (Fig. 2) of multimodality imaging studies are very heterogeneous. The part of CT in a PET/CT examination conducted as a full diagnostic CT with or without intravenous contrast enhancement is presently the practice in a relatively small number of facilities (Fig. 3). The majority of respondents of both societies believe that the proportion of PET/CT conducted as a full diagnostic CT examination, employing intravenous contrast enhancement will increase over time (Fig. 4). As expected, oncology is the most common indication for PET or PET/CT (Fig. 5), and 18F-FDG is the most used PET tracer for clinical applications (Table 3). The large majority of the respondents of both societies were in favour of an interdisciplinary

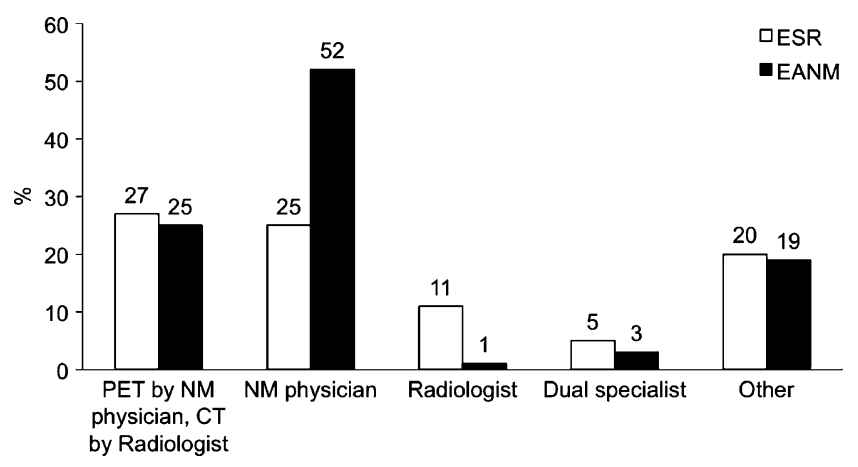

Fig. 2 Answers to question 2 of the survey, "In your facility, PET/CT results are billed by..." 


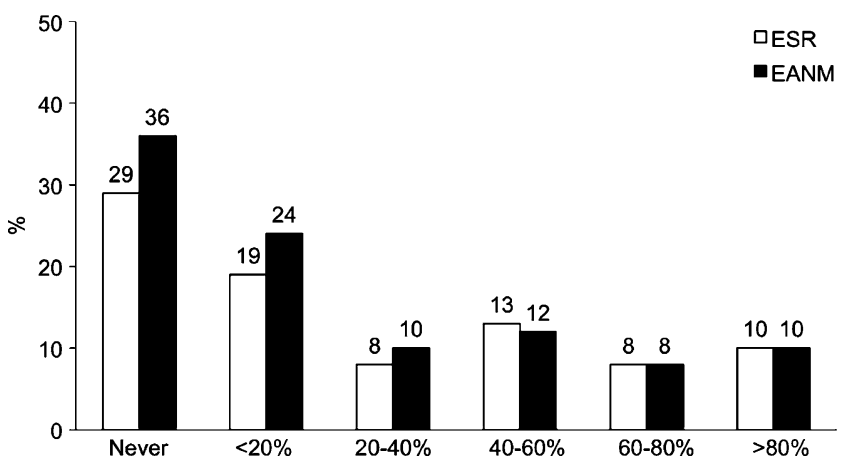

Fig. 3 Answers to question 3 of the survey, "How often is the CT part of PET/CT conducted as a full diagnostic $\mathrm{CT}+/-$ contrast enhancement in your facility?"

training programme, being developed on a European level together by ESR and EANM and the respective sections of the European Union of Medical Specialists (Fig. 6). The majority (65\%) of ESR members in favour of the principle of the interdisciplinary training programme indicated incorporated training in nuclear medicine and radiology as their preferred option (Fig. 7). The remaining 35\% favoured an adjusted period of training in the other specialty in addition to full training in the primary specialty. Among the EANM members in favour of an interdisciplinary training programme, $50 \%$ indicated as preferred option an adjusted period of training, and $50 \%$ an incorporated training in nuclear medicine and radiology taking the form of a crossover or integrated training programme (Fig. 7).

\section{Discussion}

The main purpose of this survey was to collect information on the practice of multimodality imaging in Europe among the individual memberships of ESR and EANM. It sought

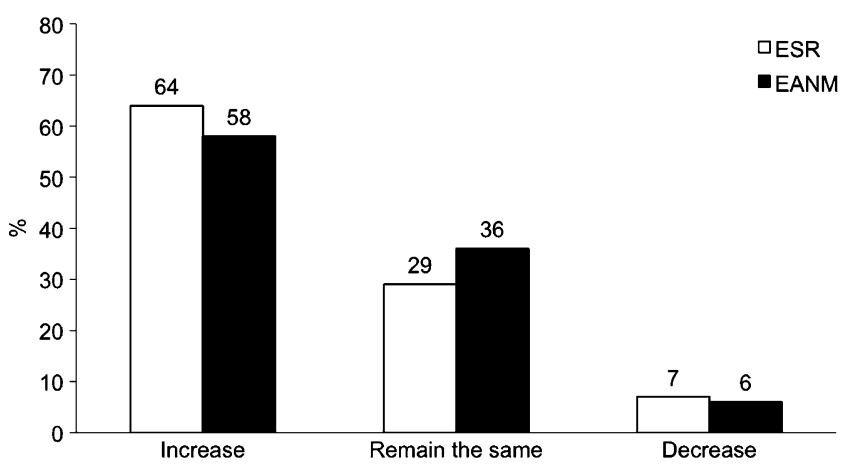

Fig. 4 Answers to question 4 of the survey, "Do you think that the proportion of diagnostic $\mathrm{CT}+/-$ contrast enhancement will...?"

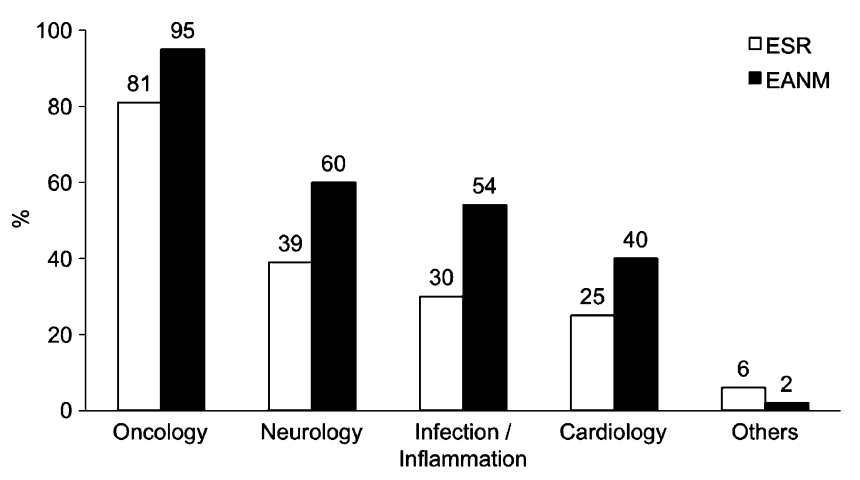

Fig. 5 Answers to question 5 of the survey, "Does your facility undertake PET or PET/CT in the following indications?"

to address regulatory and supervisory issues, the training requirements applicable and the comparative roles of radiologists and nuclear medicine physicians in the various jurisdictions surveyed.

The general limitations of a pan-European questionnaire must be taken into account when interpreting the results. This is particularly so when respondents are chosen from two independent and fully functioning disciplines as is the case in this effort. Amongst other factors the questionnaire value is dependent upon question validity and lack of ambivalence, and on the care used and bias of respondents. A certain amount of the reported data may thus not be representative, but alternative methods of gathering information in such a context are difficult, laborious and not practicable.

The majority of the members of ESR and EANM believe that the proportion of PET/CT conducted as a full diagnostic CT with contrast enhancement will increase in the future. Substantive training in both radiology and nuclear medicine is

Table 3 Answers to question 6 of the survey: radiopharmaceuticals in clinical use in European countries.

\begin{tabular}{lll}
\hline & ESR & EANM \\
\hline${ }^{18}$ F-Fluoro-2-deoxy-D-glucose (FDG) & $73 \%$ & $99 \%$ \\
${ }^{18}$ F-Dopa & $10 \%$ & $29 \%$ \\
${ }^{18}$ F-Fluorocholine & $10 \%$ & $23 \%$ \\
${ }^{18}$ F-Fluoroethyl-L-tyrosine (FET) & $5 \%$ & $12 \%$ \\
${ }^{18}$ F-Fluorothymidine (FLT) & $4 \%$ & $14 \%$ \\
Other ${ }^{18} \mathrm{~F}$ & $4 \%$ & $12 \%$ \\
Any ${ }^{11} \mathrm{C}$ & $2 \%$ & $14 \%$ \\
Rubidium-82 & $1 \%$ & $6 \%$ \\
${ }^{15 O}$-Water & $1 \%$ & $11 \%$ \\
Others & $7 \%$ & $13 \%$ \\
\hline
\end{tabular}



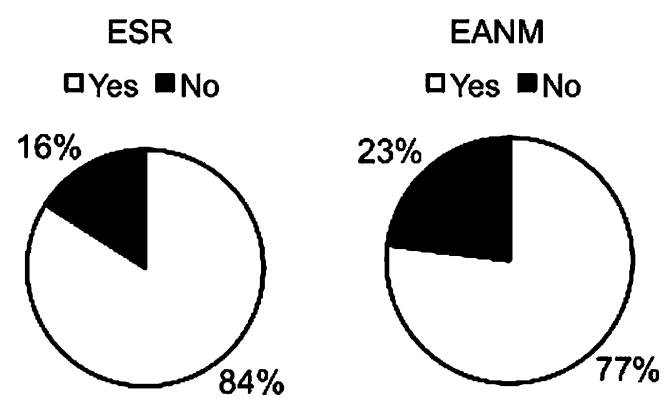

Fig. 6 Answers to question 6 of the survey, "Which of the following radiopharmaceuticals are in clinical use in your country?"

a prerequisite for high-quality examination interpretation with hybrid systems. There are several ways to achieve such training, and appropriation of "dual training" structures will differ between countries relating to differences in infrastructure and legislation $[1,2]$. In addition to being structured and comprehensive, training should be conducted in accredited training centres. It should incorporate the principles and major modalities of both specialties to allow the trainee to learn the appropriate place of PET-SPECT/CT in the overall investigative algorithm.

\section{Conclusion}

The overall findings of this survey show that there is a wide heterogeneity in the use and practice of multimodality imaging in Europe. This situation may well limit the application of the technique with its full clinical potential. As clearly indicated by the results of the present survey, the large majority of respondents of both societies were in favour of an interdisciplinary training programme, being developed on a European level together by ESR and EANM and the respective sections of the European Union of Medical Specialists.

\section{ESR}

Adjusted period of training

Integrated training programme

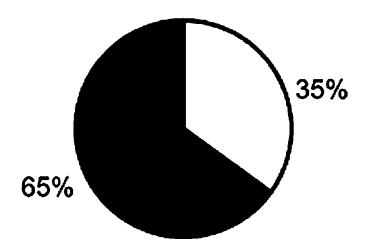

\section{EANM}

Adjusted period of training

Integrated training programme

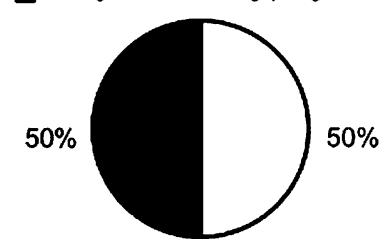

Fig. 7 Answers to question 7 of the survey, "Would you be in favour of an interdisciplinary training programme being developed on a European level together by ESR and EANM and the respective UEMS sections?"
Acknowledgement This paper from the ESR and the EANM was prepared by Drs A. Cuocolo and E. Breatnach. It is being published concurrently in the European Journal of Nuclear Medicine and Molecular Imaging Multimodality imaging in Europe: a survey by the European Association of Nuclear Medicine (EANM) and the European Society of Radiology (ESR). Cuocolo A, Breatnach E. Eur J Nucl Med Mol Imaging. 2010 Jan;37(1):163-7.

\section{Appendix}

Survey questions

1. In your facility, PET/CT results are reported by

- PET by a nuclear medicine specialist, CT by a radiologist

- A nuclear medicine physician

- A radiologist

- A dual specialist

- Other

2. In your facility, PET/CT results are billed by

- PET by a nuclear medicine physician, CT by a radiologist

- A nuclear medicine physician

- A radiologist

- A dual specialist

- Other

3. How often is the CT part of PET/CT conducted as a full diagnostic $\mathrm{CT}+/-$ contrast enhancement in your facility?

$$
\begin{aligned}
& -\quad \text { Never } \\
& -\quad<20 \% \\
& -\quad 20-40 \% \\
& -\quad 40-60 \% \\
& -\quad 60-80 \% \\
& -\quad>80 \%
\end{aligned}
$$

4. Do you think that the proportion of diagnostic $\mathrm{CT}+/-$ contrast enhancement will

- Increase

- Remain the same

- Decrease

5. Does your facility undertake PET or PET/CT in the following indications?

- Oncology

- Cardiology

- Neurology

- Infection/inflammation

- Others

6. Which of the following radiopharmaceuticals are in clinical use in your country?

- 18F-FDG 
- 18F-Dopa

- 18F-Fluorocholine

- 18F-FET

- 18F-FLT

- Other $18 \mathrm{~F}$

- Any 11C

- Rubidium-82

- 15O-Water

- Others

7. Would you be in favour of an interdisciplinary training programme being developed on a European level together by ESR and EANM and the respective UEMS sections?

- Yes

(a) Adjusted period of training in the other speciality in addition to full training in the primary speciality (b) Integrated training programme: an incorporated training in nuclear medicine and radiology taking the form of a cross-over or integrated training programme

$-\quad$ No

\section{References}

1. Gourtsoyiannis N, McCall I, Reiser M, Silberman B, Bischof Delaloye A, Carrió I et al (2007) White Paper of the European Society of Radiology (ESR) and the European Association of Nuclear Medicine (EANM) on Multimodality Imaging. Eur Radiol 17:1926-1930

2. Bischof Delaloye A, Carrió I, Cuocolo A, Knapp W, Gourtsoyiannis N, McCall I et al (2007) White Paper of the European Association of Nuclear Medicine (EANM) and the European Society of Radiology (ESR) on multimodality imaging. Eur J Nucl Med Mol Imaging 34:1147-51 